\title{
ITALIANI PER VOCAZIONE: VOCI MIGRANTI IN CERCA DI OSPITALITÀ
}

\section{ELENA BENELLI}

\begin{abstract}
This article explores the evolution of Italian migrant writing and its characteristics and analyzes the work of a young migrant writer, Jadelin Gangbo, who, with the novel Rometta e Giulieo, destabilizes the frontiers of fiction and the literary canon. The writer's identity and the borders of fiction are redefined in this modern parody of Shakespeare where the narrator enters the world of his fictional characters. The role of migrant writing as a possible hospitable site in order to elaborate a new poetic and practice of hospitality is also examined.
\end{abstract}

A partire dagli anni Novanta del secolo scorso, in Italia si sviluppa un nuovo fenomeno letterario, quello degli scrittori migranti ${ }^{1}$, che, almeno all'inizio, ha interessato grandi case editrici, e che è stato, in questi ultimi anni, analizzato dalla critica, tra molte difficoltà, dovute soprattutto al problema della definizione, del significato e del valore da attribuire agli scrittori e alle opere coinvolte. L'interesse di alcune grandi case editrici, come ad esempio la Garzanti e la De Agostini ${ }^{2}$, era dovuto ad un fenomeno nuovo per l'Italia: a causa di numerosi fatti di cronaca che registravano l'arrivo di una presunta invasione di

\footnotetext{
Termine generale utilizzato per definire questi scrittori, che in realtà sfuggono ad ogni definizione restrittiva perché, come si vedrà più avanti, hanno una scrittura molto complessa e molto variegata.

2 Hanno pubblicato rispettivamente: Pap Khouma - Oreste Pivetta, Io venditore di elefanti. Una vita per forza tra Dakar, Parigi e Milano, Garzanti, Milano, 1990 e Saidou Moussa Ba - P.A. Micheletti, La promessa di Hamadi, De Agostini, Novara, 1991.
} 
massa, per la prima volta, la penisola si è trovata a diventare il luogo di approdo di migliaia di migranti alla ricerca di un destino migliore. Se, da una parte, questo fenomeno globale ha colto tutti di sorpresa, costringendo più di un governo a fare $\mathrm{i}$ conti con strutture fatiscenti $\mathrm{e}$ leggi di emergenza, dall'altra è sembrato che un lapsus collettivo avesse sorpreso la società italiana, la quale aveva facilmente dimenticato che fino agli anni '70 erano stati gli italiani stessi a migrare in massa verso destinazioni lontane alla ricerca di una vita migliore.

Nello stesso periodo, come ho detto, vengono pubblicate alcune opere scritte da autori immigrati in italiano aventi come sfondo l'Italia, che diventa, per la prima volta nella sua storia, società di accoglienza. Mi riferisco in maniera particolare ai romanzi Immigrato di Salah Methnani e Mario Fortunato (Methnani 1990), e Io venditore di elefanti. Una vita per forza tra Dakar, Parigi e Milano di Pap Khouma e Oreste Pivetta (Khouma 1990). Questa prima fase della letteratura di migrazione è caratterizzata da una serie di elementi che Armando Gnisci è stato il primo a sottolineare ${ }^{3}$ : in primo luogo si tratta di opere scritte a quattro mani, in cui allo scrittore migrante si affianca spesso un giornalista italiano che fa da editore al testo; inoltre, questi romanzi hanno come tema principale l'esperienza autobiografica della migrazione dell'autore; infine essi vengono pubblicati, almeno all'inizio, da case editrici di successo, tra cui Garzanti, che probabilmente intuiscono i profitti e l'interesse di pubblico nei confronti di un argomento così scottante.

Entrambi i romanzi, che hanno avuto un discreto successo di pubblico, raccontano il viaggio, l'arrivo e le difficoltà insite nel trasferimento e nella sistemazione in Italia, attraverso aneddoti, storie e vicende personali degli autori alle prese con la società italiana che si ritrova del tutto impreparata e fondamentalmente ostile all'arrivo di

\footnotetext{
Per esempio in Il rovescio del gioco. Roma: Sovera, 1993, e La letteratura italiana della migrazione. Roma: Lilith (1998). Armando Gnisci ha studiato con attenzione le opere degli scrittori migranti sin dall'inizio contribuendo alla loro diffusione.
} 
queste persone. I due libri sono caratterizzati dal disorientamento dei loro autori-narratori di fronte a un nuovo paese e, come molti romanzi di migrazione, ruotano attorno all'argomento principale della ricerca non solo di un luogo ma anche di un'identità da parte dell'autore migrante.

È stato riconosciuto che nelle letterature di migrazione "a useful starting point for the discussion of the representational outcomes of migration experiences lies in setting up a conceptual framework consisting of a series of possible shifts in identity that occur in relation to migration, both at the individual and at the larger-group levels" (White 1995:2). Il paradigma identitario come un orizzonte fisso, immobile, immutabile, componente specifica dell'appartenenza a un paese, viene destabilizzato dalla scrittura di questi autori, che è in grado di indagare una realtà molto più sfaccettata e complessa. Parlando del complesso tema dell'identità, Stuart Hall (1997:47) ha dichiarato che "identities are never completed, never finished", sottolineando nel suo lavoro critico come l'identificazione identitaria si sviluppi e si modifichi attraverso molteplici esperienze e mettendo in luce che l'identità non è un dato immutabile, come alcuna propaganda politica vorrebbe far credere. Gli autori migranti articolano dunque in modo nuovo l'identità italiana problematizzandola, sottolineandone il carattere narrativo e finzionale nel momento delicato dell'incontro con l'Altro, portatore di lingue, culture e costumi diversi. In molti dei romanzi della migrazione, uno dei temi centrali è quello dell'identità: l'identità del migrante, da quella di partenza, a quella nuova, acquisita in seguito al soggiorno nel nuovo paese, quella che il protagonista percepisce di se stesso, quella che gli altri attribuiscono ai protagonisti, di solito molto approssimativamente, quella che l'individuo assume poi una volta tornato nella madrepatria, se vi torna, ed infine quella che ogni individuo si sceglie attraverso i processi che lo portano ad essere se stesso. A queste molteplici e complesse identità si può aggiungere anche 1'"identità narrativa", quella che Paul Ricoeur definisce come "la sorte d'identité à laquelle un être humain accède grâce à la 
médiation de la fonction narrative" (Ricoeur 1988:295). Grazie a questa definizione è possibile analizzare le complesse sfaccettature del problema identitario e indagare la sua narrativizzazione, da una parte per osservare i discorsi che vengono tramandati nei confronti di un concetto inteso tradizionalmente come immutabile, dall'altra invece per decostruire ogni paradigma identitario al fine di osservare come le identità contemporanee si costruiscano, perché, come ha scritto Francesco Remotti nel suo interessante saggio Contro l'identità, "l'identità viene sempre, in qualche modo, 'costruita' o 'inventata" (Remotti 1996). Remotti mette in luce nel suo lavoro soprattutto le dinamiche identitarie indagate dal punto di vista antropologico, cogliendone soprattutto l'aspetto fittizio. Egli afferma:

Se l'identità è costruita, se l'identità è una 'finzione', si impone la necessità di cogliere una logica di come è costruita: e questo compito si svolge meglio non già condividendone i principi, adattandone le modalità, facendo propria la 'finzione', indossando insomma la maschera dell'identità, bensì cogliendo principi, modalità, regole, significati, con un atteggiamento in un certo senso 'oppositivo', 'contrastante', 'a ritroso'. (Remotti 1996: 65)

Per fare ciò, oltre al livello della costruzione (discorsiva ma non solo) dell'identità, bisogna ricordare che a esso si somma un livello chiamato intermedio da Remotti, ovvero quello delle "connessioni, caratterizzato da potenzialità, ovvero da elementi alternativi" a cui si aggiunge la dimensione del flusso e del mutamento: "esso si presenta come un mutamento continuo, oscuro e magmatico, radicalmente destruttivo" (Remotti 1996:9). Introducendo il flusso e il movimento nella rappresentazione dell'identità, Remotti aiuta a comprenderla non soltanto come qualcosa di non deciso una volta per tutte, bensì soggetto al mutamento costante di cui l'identità narrativa può darci uno spaccato, narrando l'identità nel suo farsi. 
L'identità italiana, in particolare, ha un enorme debito nei confronti della narrativa perché è grazie alla narrazione, in questo caso specificatamente letteraria, che si è costruita attraverso i secoli ${ }^{4}$. Non solo: grazie alla presa di parola dei migranti, essa può incominciare a incorporare significati nuovi e permettere una più complessa articolazione di se stessa, che tenga conto di tutto quello che fino ad ora era stato considerato come subalterno, aprendosi a un più fecondo discorso che possa essere terreno di incontro e di ospitalità.

Lo scontro con la società di destinazione che questi autori subiscono all'arrivo in Italia si riflette nella perdita della loro identità di partenza, con la spiacevole conseguenza di trovarsi addosso l'etichetta che la gente ha voluto attaccare loro: quella di immigrato. In italiano esistono molte espressioni per indicare gli immigrati, e tutte possiedono una connotazione negativa. Tra queste c'è extracomunitario, che significa, evidentemente, una persona non appartenente alla comunità europea, termine che non viene mai utilizzato per indicare turisti nordamericani o giapponesi, quasi a voler simbolizzare un'esclusione a carattere economico più che nazionale. Tra gli altri termini, esiste anche il più curioso vu' cumprà che deriva dalla contaminazione del francese vous con la forma del verbo volere, vuoi, apparentemente mal pronunciata dai venditori ambulanti che circolano spesso sulle spiagge della penisola. Poi c'è marocchino, etichetta comune per tutti gli immigrati, poco importa la loro provenienza, associato anche alla rappresentazione negativa dei soldati marocchini (e nordafricani) sbarcati in Italia verso la fine della Seconda Guerra Mondiale, accusati di numerosi stupri. Questa è una semplificazione estrema per indicare una persona di origini africane che non tiene conto della ricchezza etnica del continente africano né

\footnotetext{
$\mathrm{Su}$ questo argomento, è possibile consultare: AA.VV. Italian cultural studies. An introduction. A cura di David Forgacs e Robert Lumley. Oxford: Oxford University Press, 1996. Inoltre vedere anche Remo Ceserani, Italian literary studies and the new passwords: prospectivism, polysystem, comparatism. Remapping the boundaries. A new perspective in comparatives studies. A cura di Giovanna Franci,. Bologna: Clueb, 1997:39-46. Infine il volume di Ernesto Galli della Loggia L'identità italiana. Roma: Il Mulino, 1998.
} 
della complessità di ogni singola nazionalità, al cui interno esistono tradizioni diverse. Pap Khouma, l'autore di Io venditore di elefanti, ha aiutato una squadra di volontari italiani a scrivere un libro: Nato in Senegal, immigrato in Italia (Khouma 1994), in cui, in una breve scheda introduttiva sul Senegal, spiega che nel suo paese esistono diverse lingue, di cui gli italiani ignorano l'esistenza e che ridurre $\mathrm{i}$ senegalesi a dei marocchini equivale a commettere non solo un errore geografico ma una reductio ad unum estremamente semplicistica. Un altro termine che viene utilizzato è zingaro, se si tratta di popolazioni nomadi di qualunque provenienza, o infine, clandestino. Quest'ultimo, paradossalmente democratico, vale per tutti.

Il processo di identificazione dei migranti con termini negativi ha un doppio valore. Da definizione riduttiva, esso si carica di un ulteriore significato, nel senso che gli immigrati che subiscono questa etichettatura possono decidere a loro volta di comportarsi secondo queste categorie per rispondere alle aspettative degli italiani. Per la prima volta, questi scrittori si ritrovano addosso un'etnia e un'etichetta di cui prima non erano affatto consci. Stuart Hall spiega il processo che lo ha portato a riconfigurare più di una volta la propria identità nel momento in cui si trovò ad immigrare in Inghilterra: "I became what I have been named. I had been nailed as an immigrant. I had discovered who I was. I started to tell the story of my own migration. Then Black erupted and people said [...] 'You're Black"' (Hall 1997:55). L'autore incomincia a scoprire l'esistenza di una nuova categoria, the Black category, che soppianta quella semplicistica di immigrati, nello stesso modo in cui gli immigrati in Italia scoprono quella onnicomprensiva di extracomunitari: una categoria che etnicizza il soggetto e lo rinchiude in un'identità precostituita che non corrisponde alla realtà ma ai pregiudizi di chi ne fa uso.

Una seconda fase di scrittura incomincia qualche anno dopo, a metà anni Novanta, quando gli autori migranti pubblicano senza l'ausilio del co-autore italiano poiché scrivono direttamente in italiano. Questa scelta non è di poco conto: da una parte, essa indica l'assimilazione e la padronanza della lingua mentre dall'altra l'italiano 
diventa per la prima volta una lingua "neutra" appartenente a una comunità internazionale. L'italiano è una lingua neutra per la maggior parte di questi scrittori perché non legata al passato coloniale, come possono esserlo l'inglese o il francese. Gli autori migranti che scrivono in Francia provengono da ex-colonie in cui il francese è la lingua del colonizzatore ${ }^{5}$. Nel caso dell'italiano, gli autori la assimilano come seconda o terza lingua, se consideriamo come prima la madrelingua e come seconda la lingua del paese colonizzatore. Ciò non esclude il caso di autori che scrivono in italiano provenienti dalle ex-colonie italiane, Somalia o Eritrea ad esempio, ma bisogna sottolineare che il sintomo della scelta dell'italiano va al di là del dibattito post-coloniale. Gli autori migranti in Italia provengono dai paesi più disparati, e questo fattore contribuisce a creare una letteratura migrante in italiano che è un caso unico al mondo. Essi sono albanesi, serbi, croati, senegalesi, brasiliani, somali, iraniani, russi, polacchi e di altre nazionalità ancora, senza che ce ne sia una predominante; anche questo fattore li distingue dagli altri autori dei paesi post-coloniali in cui spesso gli autori migranti appartengono a una regione ben precisa con cui il paese d'accoglienza intrattiene rapporti neo-coloniali dopo la rottura del legame antecedente. Come ha sottolineato Julio Monteiro Martins,

L'Italia non ha avuto una letteratura post-coloniale ricca come la Francia o l'Inghilterra. Per questo le è mancato un flusso letterario importante ma forse ha lasciato questo territorio libero, in modo che, non a caso a parer mio, l'Italia è diventata il centro della letteratura di migrazione in Europa. Credo che da quanto ho letto in nessun altro

\footnotetext{
Per esempio Tahar Lamri, uno scrittore di origine algerina, ha dichiarato su El Ghibli: "Per me, scrivere in Italia, paese dove ho scelto di vivere e con-vivere, vivere nella lingua italiana, convivere con essa e farla convivere con le altre mie lingue materne (il dialetto algerino, l'arabo ed in un certo senso il francese) significa forse creare in qualche modo l'illusione di avervi messo radici. Radici di mangrovia, in superficie, sempre sulla linea di confine, che separa l'acqua dolce della memoria, da quella salata del vivere quotidiano". Lamri Tahar, Il pellegrinaggio della voce, El Ghibli 2 (2003). http: //www.elghibli.provincia.bologna.it/id_1-issue_00_02-section_6-index_pos_3.html.
} 
paese, forse il numero degli autori è lo stesso, ma non nella diversità delle origini degli scrittori che qua è un ventaglio impressionante. Non sarà che il fatto di non aver avuto una letteratura post-coloniale abbia favorito il proliferare di questa letteratura mondiale, viaggiante? (Martins 2005)

Questo cantiere umano così ricco e variegato offre alla letteratura migrante in lingua italiana una ricchissima varietà di apporti sia linguistici, che letterari e culturali. La rende un bacino prezioso di forme, idee, strutture che possono arricchire la letteratura nazionale che ha da tempo bisogno di rinnovamento. Nonostante questo dinamismo e il moltiplicarsi delle iniziative di promozione da parte di circoli culturali locali, le grandi case editrici, dopo l'interesse iniziale, sono restate indifferenti a queste opere che vengono stampate da piccoli editori, che fanno spesso fatica a trovare un distributore, per cui spesso vengono vendute per strada da ragazzi immigrati alla ricerca di una possibile integrazione. ${ }^{7}$ Armando Gnisci ha definito questo momento della letteratura italiana della migrazione una fase carsica: "è proprio in questo momento cruciale che la letteratura italiana della migrazione ha svoltato e ha imboccato la sua strada autentica e fruttifera, abbandonata a se stessa non si è disseccata ma ha trovato da vero fiume con una propria corrente una specie di vena carsica" (Gnisci 1988:42). Lo stesso Gnisci, professore di letteratura comparata all'università di Roma, è stato uno dei promotori di queste opere: grazie ai suoi numerosi contributi critici e alla creazione di una rivista di letteratura di migrazione on line, Kúmá.Creolizzare l'Europa ${ }^{8}$, finanziata dall'università stessa, ha grandemente

\footnotetext{
All'indirizzo http: //www.sagarana.net la rivista on line Sagarana mette a disposizione gli atti del congresso annuale.

7 Per esempio la Sinnos di Roma, Sensibili alle foglie sempre a Roma ad opera di Renato Curcio, Fara editore a Sant'Angelo di Romagna, edizioni dell'Arco a Milano.

Http: //www.disp.let.uniroma1.it/kuma/kuma.html.
} 
contribuito alla diffusione di questi testi e di questi autori. In effetti, la rete, come veicolo di distribuzione democratico delle informazioni, è stata quella che ha permesso a molte opere di circolare e di diffondersi ${ }^{9}$.

Tra gli autori di questo periodo carsico, alcuni di essi risiedono in Italia da parecchi anni, come Kossi Komla-Ebri ${ }^{10}$ che ha pubblicato un romanzo $^{11}$ e $\mathrm{i}$ divertenti e istruttivi racconti brevi dal titolo Imbarazzismi (Komla-Ebri 2002 e 2004) in due volumi successivi. Possiamo aggiungerne molti altri tra cui lo stesso Julio Monteiro Martins $^{12}$ e Pap Khouma, che non solo è redattore di una rivista che si occupa di letteratura migrante ma ha anche pubblicato un secondo romanzo ${ }^{13}$. A questi si sommano autori vissuti tutta la vita in Italia, $\mathrm{i}$

9 Tra le iniziative on-line va ricordata una rivista on-line ad opera del già citato Pap Khouma, El-Ghibli (http: //www.el-ghibli.provincia.bologna.it/) sponsorizzata dalla provincia di Bologna. La rivista è un autentico contenitore di poetiche, scritture, idee da parte di autori migranti e non, in cui l'identità multipla trova uno spazio per esprimersi e per uscire dai confini canonici in cui la si vorrebbe relegare. Queste iniziative permettono un grande accesso di pubblico a costi più bassi di quelli editoriali tradizionali, mentre, allo stesso tempo, promuovono alacremente e con gran volontà $i$ testi degli scrittori migranti nonché dibattiti e incontri culturali. Un altro sito internet di grande utilità è il sito sponsorizzato dal comune di Ferrara, Voci dal silenzio (http: //www.comune.fe.it/vocidalsilenzio/index.htm) che mette a disposizione un buon numero di materiale di ricerca on line. Infine, dobbiamo ricordare l'associazione culturale Eks\&Tra (http: //www.eksetra.net) che, come viene spiegato nell'introduzione al sito: "il nome Eks\&Tra che abbiamo scelto per presentarci indica la provenienza da altri paesi: Eks=ex, e l'arrivo Tra noi. L'\& è una congiunzione che assomma in sé le difficoltà e insieme la grande ricchezza dell'incontro. Il premio Eks\&Tra è nato nel 1995 a Rimini e si è trasferito a Mantova nel 1999 grazie all'interessamento del Centro di Educazione Interculturale della Provincia di Mantova". (http: //www.eksetra. net/associazione/descrizione.shtml) On line inoltre si trovano anche le edizioni del concorso con quasi tutti gli interventi critici delle varie edizioni. L'associazione inoltre promuove un concorso letterario annuale che ha permesso a molti scrittori di farsi conoscere, i quali grazie a questa iniziativa, hanno trovato editori disposti a pubblicare le loro opere. Kossi è stato per parecchio tempo collaboratore per il giornale Repubblica, per il quale ha tenuto un blog, e oggi scrive e pubblica anche sul proprio sito internet: http: //www.kossikomlaebri.net/index.php.

11 Neyla, Incontro tra due mondi (Komla-Ebri 2002).

12 Racconti italiani (Martins 2000). Inoltre Martins ha pubblicato abbondantemente in Brasile.

13 Nonno Dio e gli spiriti danzanti (Khouma 2005). 
quali hanno frequentato scuole italiane e che considerano l'italiano come la loro madre lingua. Si può ricordare soprattutto Igiaba Scego che ha pubblicato due romanzi ${ }^{14}$ e una raccolta di racconti a cui ha fatto da editore. Questi autori migranti vengono indicati come hyphenate writers $^{15}$ in Nord America, dove sono demarcati dal trattino di congiunzione tra le due designazioni etniche. Anthony Tamburri ha definito questo trattino come "an ideologically charged marker" (Tamburri 1991:11) perché costituisce un segno distintivo che carica il primo elemento di subalternità (come per esempio in ItalianAmerican). Ci troviamo d'accordo con Tamburri, dal punto di vista teorico, sulla scelta del segno da utilizzare da lui proposta, ovvero non più il trattino, -, l'hyphen, ma bensì la barra obliqua, /, lo slash in inglese, che non divide ma unisce in un'alternanza altamente problematica due nazionalità, due costrutti storici e culturali, che non restano separati, ma creano a loro volta una nuova forma ibrida di identità nazionale.

Come definire dunque questi scrittori? Da una parte chiamarli migranti sottolinea le loro origini, ma dall'altra essi stessi sono contrariati dell'essere rinchiusi nel ghetto di una definizione la quale, sebbene possa aiutare all'inizio a prendere la parola, dall'altra rischia di diventare come una sorta di gabbia in cui essere rinchiusi. ${ }^{16}$

14 I romanzi sono: La nomade che amava Alfred Hitchcock (Scego 2003) e Rhoda (Scego 2004), e la raccolta porta il titolo Italiani per vocazione (Scego 2005).

15

Il termine hyphenate è stato usato per la prima volta da Daniel Aaron nel 1964 nell'articolo The hyphenate writer and American letters, Smith Alumnae Quarterly, July 1964:213-217 e, secondo Anthony Tamburri che lo cita nel suo To hyphenate or not to hyphenate (Toronto: Guernica, 1991:28), egli afferma che "the hyphen initially represented older North Americans' hesitation to accept the new/comer; it was their way [...] to 'hold him at 'hyphens' length', so to speak, from the established community". Il trattino designa dunque sin dall'inizio la distanza che la società d'accoglienza vuole mantenere con l'immigrato.

16 Jadelin Gangbo in un'intervista ha dichiarato: "Tutti aspiriamo ad una realtà ufficiale, candida, asettica quale quella della letteratura italiana. Ma dinamiche editoriali ci respingono fuori da tale canone italianissimo. Io sono contento di essere stato respinto, perché è proprio grazie a questo rifiuto che ho capito quale sia il vero potenziale della scrittura mia e degli altri colleghi migranti: la molteplicità. Noi siamo qui e altrove allo stesso tempo. Italiani e 
Questa situazione poco canonica inserisce questi scrittori in una posizione al margine della letteratura ufficiale che, nella maggior parte dei casi, li snobba. Eppure essi rivendicano il diritto alla scrittura anche grazie a questa posizione periferica che li distanzia tanto dal discorso ufficiale, quanto dal ghetto dello scrittore per forza "migrante". Essi si muovono su una sottile linea di in bilico tra la nazionalizzazione e l'esclusione. Vivono il paradosso di essere italiani pur essendo altri, stranieri, di origine e influenze diverse, e di essere altri, pur essendo italiani, spesso non solo di adozione.

Tra questi scrittori, mi soffermo su uno decisamente originale, Jadelin Gangbo, di origine congolese, trasferitosi in Italia da bambino con la propria famiglia da cui è stato separato dopo la fuga e il tracollo finanziario del padre. Egli ha vissuto e studiato in Italia ed è autore di due romanzi: Verso la notte Bakonga (Gangbo 1999) e Rometta e Giulieo (Gangbo 2001), quest'ultimo pubblicato da Feltrinelli.

Rometta e Giulieo è un'operazione originale di riscrittura del classico shakespeariano, definita romanzo benché si tratti di un'opera ibrida che potrebbe benissimo essere un testo teatrale ${ }^{17}$; essa innova il canone letterario: un autore migrante riscrive un testo classico, il Giulietta e Romeo di Shakespeare e non solo ne imita la trama ma, in

non allo stesso tempo". Scego, Migranti o vincenti ma scrittori; dal link http: //italy.peacelink.org/migranti/articles/art_15369.htm.

Sullo stesso tono la Scego che ha lo stesso atteggiamento di Gangbo: "Non mi piacciono le etichettature, perché quando penso alla scrittura migrante io penso a una scrittura che parla di immigrazione, ma io personalmente non vorrei limitarmi a questo. Credo che gli autori cosiddetti migranti, che provengono da altre parti del mondo, non vogliano limitarsi a scrivere soltanto di immigrazione. Trovo che sia una gabbia dover parlare di emigrazione. Io vorrei parlare sia d'emigrazione che è giustissimo, ma anche d'altro, e invece noi autori di origine non italiana siamo ingabbiati dalle nostre origini. Questa limitazione è molto forte, anche le case editrici che si avvicinano a noi, sono case editrici che si occupano di intercultura, ma penso che se noi sorpassiamo questo limite della intercultura, potremo trovare qualcuno che ci pubblica". Maria Cristina Mauceri, "Igiaba Scego: la seconda generazione di autori transnazionali sta già emergendo," El Ghibli 4 (2004).Http://www.el-ghibli.provincia.bologna.it/id_1-issue_01_04-section_6-index_pos_ 1.html.

17 Infatti il romanzo è stato adattato alla scena nel 2004 da Arena del Sole, Nuova Scena del Teatro stabile di Bologna in collaborazione con il teatro stabile di Brescia con regia di Abdherrahim El Hadiri. 
alcune parti del romanzo, ne prende in prestito lo stile, mescolando l'italiano standard, colloquiale e parlato, a un italiano aulico, con un effetto decisamente straniante; infine la tecnica narrativa confonde $i$ mondi finzionali ed è accentrata attorno a una metalessi, una figura retorica che secondo la definizione dataci da Gérard Genette consiste nel narrare cambiando livello. Il critico francese spiega che "questi giochi narrativi manifestano con l'intensità dei loro effetti l'importanza del limite che essi si ingegnano a superare a scapito della verosimiglianza coincidente proprio con la narrazione $\mathrm{o}$ rappresentazione stessa: frontiera mobile ma sacra fra due mondi: quello dove si racconta, quello che si racconta" (Genette 1976:282; corsivo mio).

Il romanzo è stato pubblicato nel 2001 presso Feltrinelli, il che ha permesso una buona diffusione dell'opera nonché una certa visibilità che purtroppo non è durata molto ${ }^{18}$. Graziella Parati lo ha definito come "a new model for post-migrations and post-ethnic writings. Through the use of irreverence, breaking linguistic codes, and entering in to the realm of Italian literature, Rometta e Giulieo conclusively challenges the limitations inherent in defining migration literature in Italian as Italophone literature and is a provocative text for the development of Italian destination cultures" (Parati 2005:82) ${ }^{19}$. Poiché il testo di Gangbo si trasforma in un crocevia di altri testi, di referenze, e di livelli narrativi, esso è un'opera complessa e articolata che ha moltissimi richiami intra e extra testuali per cui l'etichetta di letteratura della migrazione è limitativa. Per tornare al romanzo, si può

18 Infatti ormai il romanzo è di difficile reperimento, pur essendo un romanzo molto complesso che, se fosse stato pubblicato da u autore "italiano" avrebbe molto probabilmente avuto un'altra sorte.

19 Per la studiosa, una destination culture è "the context in which terms such as migrant writer, post-migrant literature, and post-ethnic identity will be part of a more complex discourse on cultural hybridity. In a destination culture the emphasis will be on differences rather than on the construction of cultural majorities"(Parati, 2005:17). Ci troviamo d'accordo con il discorso generale effettuato dalla Parati e con molte sue considerazioni, ma ci chiediamo se una "post-ethnic culture" sia davvero una definizione attuale per l'Italia di oggi che sembra dover ancora affrontare un discorso sull'etnicità, sull'immigrazione e sull'integrazione. 
notare, fin dal titolo, uno strano capovolgimento: il tradizionale titolo shakespeariano viene sostituito da un titolo ibrido. L'autore informa già dall'inizio della propria operazione, ovvero della riscrittura dell'opera di Shakespeare. Il paratesto, definito da Genette come "une zone de transaction: un lieu privilégié d'une pragmatique et d'une stratégie, d'une action sur le public au service [...] d'une lecture plus pertinente" (Genette 1987:8), diventa qui una zona ambigua che si carica ancora di un ulteriore significato quando si osserva la copertina del libro: una foto in bianco e nero dei piedi e delle caviglie di una persona di colore in bilico sul cornicione di un terrazzo o forse un tetto, che si può presumere guardare davanti a sé nel vuoto. Di fronte ci sono alcuni tetti e gli alberi di una città qualunque. Già dalla copertina dunque siamo indotti a pensare che l'autore stesso si trova in bilico tra tradizione e innovazione, tra discorso ufficiale e alternativo, tra lingua aulica e lingua moderna. La foto diventa ancora più simbolica quando si scoprirà, avanzando con la lettura, che uno dei protagonisti del romanzo che è anche colui che lo sta scrivendo, ama salire sul cornicione del tetto del proprio palazzo per osservare il mondo circostante. Altamente drammatica arriva anche la conclusione del racconto quando il protagonista da quel cornicione spiccherà l'ultimo volo. Questo trovarsi in bilico, trovarsi sul bordo, ben rappresenta lo statuto dello scrittore migrante che si trova in un luogo inter-medio (Bhabha 1994:12) ${ }^{20}$, tra la società d'accoglienza e quella di partenza, apportando alla prima una cultura e una visione della seconda, contaminandone gli interstizi, formando nuovi spazi di dialogo.

Il romanzo è diviso in due atti: nel primo, suddiviso in venticinque capitoletti molto brevi di un paio di pagine ciascuno, viene narrata la storia di Rometta e Giulieo, i protagonisti finzionali del racconto, del loro incontro e della loro storia d'amore mentre in contemporanea il

20 Inter-medio traduce l'in-between del lavoro di Bhabha in inglese perché "gli spazi culturali non sono soltanto ubicati fra i luoghi egemonici ma rappresentano un'istanza di mediazione che risolve il gioco delle differenze attraverso una strategia oppositiva in grado di trascenderle" (dalla nota del traduttore). 
narratore racconta la propria vita e le difficoltà insite nell'operazione di scrittura di un romanzo i cui protagonisti sono proprio Rometta e Giulieo; nel secondo, composto da venti capitoletti brevi, avviene il tragico collasso della storia quando protagonisti finzionali e autore "reale" si incontrano nello stesso mondo finzionale, quello del romanzo.

Due storie si intersecano: la prima riguarda i protagonisti della storia fittizia che sono Rometta, una studentessa che all'inizio del romanzo prepara una tesina su un film di Peter Greenaway ${ }^{21}$, e Giulieo, un ragazzo cinese di professione portapizza e di vocazione ladruncolo di periferia. I due si incontrano e si innamorano non senza difficoltà, come i due amanti shakespeariani. A muovere i fili della storia invece c'è il narratore che si presenta con il proprio nome soltanto a pagina cinquantasette: "Mi chiamo Jadelin e sono un figlio di puttana" (Gangbo 2001:57). Il fatto che il narratore si chiami come l'autore del libro stesso è una sorpresa che non lascia indifferente il lettore: grazie a questo artificio che regge l'intero libro, il Jadelin personaggio ha una triplice funzione. Egli è uno dei protagonisti del romanzo, infatti lo seguiamo nelle sue peripezie e nei suoi spostamenti; allo stesso tempo è il narratore di entrambe le storie, la propria e quella dei protagonisti fittizi, ed infine è l'autore della storia che scrive davanti allo schermo del proprio computer in cui ad un certo punto del racconto entrerà.

Questo innalzamento a potenza della voce narrante complica i livelli narrativi all'interno del romanzo: Rometta e Giulieo vengono creati e raccontati dal narratore-autore Jadelin, un alter ego dell'autore vero, il quale, innamoratosi anche lui di Rometta, entra nella storia per incontrarla, sotto le mentite spoglie di Don Lorenzo, come il personaggio shakespeariano che tenterà di aiutare gli innamorati senza riuscirci. Non solo l'autore fittizio del romanzo entra nel livello narrativo dei suoi personaggi, ma i suoi personaggi entrano nel livello

21 Drowining by numbers di Greenaway, dal riferimento al personaggio Smut del film stesso, ossessionato dalla conta dei numeri. 
narrativo del loro autore in più di una occasione. Inoltre, anche il Lettore viene chiamato in causa, dato che il narratore si rivolge a lui in molteplici occasioni denominandolo "Sire", mentre lo interpella con deferenza. L'intero universo narrativo tradizionale ne esce sconvolto nel tentativo di seguire la voce narrante.

Molteplici sono i luoghi in cui si svolge l'azione: sappiamo che Jadelin vive a Bologna (come il vero scrittore); Rometta e Giulieo vivono e si incontrano a Verogna, luogo immaginario, che ricorda la ben nota città di Verona, luogo prescelto della tragedia shakespeariana, il cui nome nasce, secondo Graziella Parati dalla fusione di Verona e vergogna (Parati 2005:82) ${ }^{22}$ e che, secondo noi, è collegato con la parola rogna ${ }^{23}$; tutti i protagonisti si incontrano in un fantomatico Sud, un altro luogo senza definizione precisa in cui avviene il dramma. Tutti questi luoghi sono portatori di una realtà urbana moderna in cui si affacciano solitudine e degrado. Rometta condivide l'appartamento con alcune amiche e colleghe di università, Giulieo vive in un seminterrato prima di essere scacciato "come uno scarafaggio da sotto la credenza" (Gangbo 2001:47) e costretto a cercar riparo dalla sua amata che, a sua volta, dovrà costringerlo ad andarsene visti i litigi con le compagne; Jadelin ha un appartamento che sembra diventare sempre più sudicio mano a mano che procede nella scrittura: "uno spettacolo postatomico [...] pietoso [...] nello stesso istante uno scarafaggio mi attraversò il tavolo, Sire, e altri, con la calma dei pellegrini, risalivano il frigo [...] ebbi la netta sensazione che qualcuno mi avesse rapito la casa da un tempo indescrivibile, Sire" (Gangbo 2001:66). Giulieo lavora per una pizzeria e incontra

22 "The characters live in the city of Verogna, a name that suggests vergogna and also sounds like Verona, the setting for the Shakespearian play."

23

Rogna in italiano ha più significati: il primo e più corrente è un sinonimo per scabbia che dà luogo a dermatiti pruriginose negli esseri umani e negli animali; in botanica illustra una malattia che si manifesta in un agglomerato di escrescenze irregolari; in senso figurato significa qualcosa che procura notevoli fastidi e guai. Crediamo che quest'ultima definizione che mette in rilievo con la prima il carattere fastidioso dell'evento o della malattia, sia l'indicazione da seguire. 
Rometta in una via poco lontano dal locale mentre lui sta spingendo il motorino per farlo ripartire e le chiede aiuto. Alla fine del romanzo Giulieo cerca Rometta in una città fantasma in cui non riesce più a trovare la strada né la via della stazione, un vero labirinto. Egli è costretto a fermarsi a un distributore di benzina con la macchina del suo amico egiziano Baldassà per chiedere informazioni ma un altro cinese, un doppio di Giulieo creato apposta da Jadelin, getterà un mozzicone di sigaretta per terra, causando la morte dell'amico e cambiando per sempre la sorte dei due innamorati. Jadelin si trasforma allora in frate Lorenzo, come nella tragedia, ma invece di aiutare Romeo, nel romanzo il personaggio non è spinto da altruismo, bensì ha come secondo fine quello di conquistare Rometta e di evitare che essa ritrovi Giulieo. Rometta alla fine del romanzo si suiciderà come Giuletta nella tragedia, Giulieo invece sparirà dopo aver ucciso frate Lorenzo mentre Jadelin, pur continuando a portare sulla fronte la cicatrice della pallottola sparata da Giulieo, salterà nel vuoto dal proprio terrazzo.

È necessario sottolineare che l'operazione di riscrittura del classico si avvale di componenti originali che vengono però estrosamente elaborati. In primo luogo, entrambe le trame si svolgono in epoca contemporanea: Rometta e Giulieo vivono a Verogna, Giulieo possiede un motorino grazie al quale consegna pizze, Rometta studia Greenaway, uno sciopero dei treni ritarda la partenza di Giulieo, Giulieo ha a disposizione l'automobile di Baldassà, così come Jadelin vive inequivocabilmente nella Bologna di oggi. In netta contrapposizione temporale, i protagonisti e tutti coloro che circondano il mondo di Rometta e Giulieo si esprimono in un italiano arcaico, quasi aulico, caratterizzato dalla moltiplicazione degli aggettivi anche disusi, l'utilizzazione di vocaboli arcaici, per esempio scudi per euro, l'uso del vocativo a fine enfatico, la proliferazione dei congiuntivi esortativi, la dislocazione a sinistra di alcuni genitivi. Invece, nella parte del romanzo in cui è Jadelin il protagonista, l'italiano è l'italiano contemporaneo, lingua colloquiale, gergale, d'uso quotidiano. Quando avviene l'incontro tra questi due mondi, 
anche le lingue si scontrano: ad un certo momento, frate LorenzoJadelin deve dare spiegazione di questa strana lingua una sera a tavola insieme a Giulieo, Montecchi e Capuleti, due itineranti che dormono nella sua macchina. Infatti i due barboni gli fanno notare: "Padre, voi lo sapete che il linguaggio vostro è mutato? Vero Montecchi?" "Sì, vogliate perdonarci, Padre, ma è proprio vero, parete un'altra persona, tanto che mi stupite" (Gangbo 2001:139). A questo punto per scusarsi e per coprire la propria identità, frate Lorenzo dichiara che si tratta di un esercizio per i ragazzi che seguono un suo corso in cui devono parlare un idioma arcaico (qui l'autore fa passare per un idioma arcaico l'italiano contemporaneo). Lo scopo dell'esercizio è "di introdurre nei giovini il lato più costruttivo della menzogna in modo che essi non vadano spargendo inutili bugie per inibire altri e se medesimi, bensì riescano dalla menzogna a estrarre il lato più costruttivo. In questo modo è possibile far di una vita grigia un'altra parallela, nella quale sfogare le fantasie che nel sottile spirito non solo si annidano ma spingono, mi comprendete?" (Gangbo 2001:139). Mentre il protagonista cerca di sviare la questione, uno dei due barboni lo riprende e sottolinea con molta lucidità che il frate sta parlando della finzione. Il discorso effettuato dall'autore sottolinea la portata metanarrativa del brano che diventa una dichiarazione di poetica da parte dell'autore e che indirettamente allude al significato primo di finzione, intimamente legato da una parte alla menzogna, al dire il falso, e dall'altra alla creazione dei mondi finzionali.

L'operazione di riscrittura dell'opera shakespeariana, con i suoi molteplici livelli narrativi e numerosi registri linguistici, costituisce un'opera complessa e innovativa, importante nel panorama letterario italiano contemporaneo. Inoltre, l'utilizzo sapiente della metalessi ricorda molta fiction postmoderna. Nel caso di Rometta e Giulieo, tutto viene retto dal congegno metalettico: il narratore, che racconta tutta la storia, la propria e quella dei propri personaggi in maniera onnisciente, colloca l'intera vicenda in un lontano passato remoto a cui il Sire, ovvero il lettore, invocato numerosissime volte, deve dare un senso; i personaggi che prendono contatto con lo scrittore 
finzionale di cui cercano freudianamente di sbarazzarsi, riescono a portare a termine la loro storia senza però pervenire a modificare il loro destino di carta ma agendo su quello "reale", appartenente al livello narrativo da cui parla la voce narrante; il lettore, quello vero, deve assumere il proprio compito di interpretare il testo, rischiando sempre di trovarsi al punto di partenza perché l'invocazione continua della sua presenza non fa che riportarlo all'interno dell'aggrovigliata gerarchia narrativa.

Il romanzo di Gangbo ha una funzione pluridiscorsiva in senso bachtiniano (Bachtin 1997:71), poiché si apre alle influenze linguistiche, epocali e narrative della società italiana moderna che si avvia, volente o nolente, verso il pluralismo culturale. Se come ha scritto Alessandro Portelli, "migrant writers have claimed new territories" (Portelli 2006:474), essi agiscono in primo luogo sul territorio specifico della letteratura, modificandola dall'interno. Per tentare di capire meglio questi autori è necessario riflettere più che in termini di autori migranti, in termini di autori "minori", autori che lavorano sulla letteratura e nella letteratura direttamente dal di dentro. La letteratura minore, infatti, come l'hanno definita Deleuze e Guattari " $n$ 'est pas celle d'une langue mineure, plutôt celle qu'une minorité fait dans une langue majeure" (Deleuze 1975:29). Per questi scrittori "minori", la cui lingua non è sempre l'italiano, l'utilizzazione e la scelta dell'italiano non è solo una necessità comunicativa ma referenziale e innovativa, essa lavora l'italiano dall'interno, lo arricchisce, lo forgia a proprio modo ${ }^{24}$, si confronta con esso. Inoltre ogni testo assume un valore collettivo non in senso etnico ma poiché questi autori rivendicano gli stessi obiettivi poetici di altri autori italiani. Questi autori deterritorializzano la lingua italiana nello stesso modo in cui Kafka ha deterritorializzato il tedesco e Svevo l'italiano.

24 Ad alcuni autori è stato consigliato di lasciare nei loro testi alcuni piccoli errori nell'uso dell'italiano proprio per lasciare una traccia della loro etnicità e della loro diversità culturale, così come alcuni scelgono di usare parole nella loro lingua materna, poi chiosate a fondo pagina. 
Bisogna ricordare che è nella letteratura come sistema cognitivo che una nuova retorica dell'ospitalità può nascere. Nella metafora dell'apertura e dell'accoglienza troviamo le tracce di una pratica alla base di tutte le organizzazioni umane, quella dell'ospitalità. Il momento dell'accoglienza ospitale fonda l'incontro con l'Altro, riorganizza e ristruttura tutta la società contemporanea, la quale è sempre di più influenzata dalle dinamiche dell'incontro tra il locale $\mathrm{e}$ il globale, tra il dentro e il fuori. Nonostante la prospettiva della libera circolazione delle persone sul globo terrestre e non solo delle merci e del capitale, assistiamo invece a un irrigidimento delle frontiere dei paesi occidentali nell'accogliere tutti coloro che, estranei, diversi, e soprattutto stranieri, provengono dalla periferia dell'Impero. La frattura che caratterizza le democrazie dei nostri tempi è quella dello stato di eccezione, come Giorgio Agamben lo ha definito (Agamben 2003), che produce un'ospitalità fondamentalmente ostile ai migranti. Anna Simone, sociologa dell'università di Bari, riassume la situazione in questo paragrafo che cito per intero:

Da una parte non esiste più la dimensione unilineare della storia che escludeva le società altre in quanto statiche $\mathrm{e}$ non ancora votate al progressismo: è fuori discussione che l'altro non abita più mondi lontani ma è ormai sempre con noi. Dall'altra apre a nuovi scenari legati alla trasformazione del tempo e dello spazio in cui si costruisce l'antagonismo presente e a venire: il tempo è quello delle società globali [...], lo spazio è quello grande delle metropoli, il tutto in un affermato riconoscimento della molteplicità delle culture e della loro tendenza ad elaborare in modo eterogeneo un pluralismo antropologico in cui le nuove soggettività di lotta appartengono alla geofilosofia del nomade-migrante. (Simone 2002:36)

È innegabile che gli ultimi anni abbiano visto ridefinirsi concetti come quelli di nazione, di nazionalismo, di identità nazionale, dei centri 
nazionali e transnazionali, in cui il potere si diffonde e delle periferie in cui si riconfigura, delle frontiere e dei criteri per attraversarlo. Si tratta di idee complesse che non possono essere esaminate nel dettaglio in questa sede ma, se come afferma la Simone "l'altro è ormai sempre con noi" e le distanze tra centro e periferia sono annullate, è attraverso il terreno d'incontro con i migranti che la letteratura propone, che è necessario confrontarsi, su molti piani, non solo quello accademico. Come coloro che accolgono secondo le leggi dell'ospitalità, non solo quelle imposte dal diritto dello jus solis o dello jus sanguinis, ma in nome di un'ospitalità ideale dove il dialogo, anche letterario, diventa strumento di conoscenza reciproca. Gli scrittori migranti collaborano alla creazione di nuovi mondi, di nuovi territori, di nuove mappe, di nuovi immaginari che, almeno nella letteratura, tentano di convivere pacificamente tra loro, come ha detto Armando Gnisci: "un mondo dove molti mondi abbiano il loro posto" (Gnisci 2003:103).

(Universitè de Montréal)

\section{Bibliografia}

Agamben, G.

2003 Lo stato di eccezione. Milano: Bollati Boringhieri.

Bacthin, M.

1997 Estetica e romanzo. Torino: Einaudi.

Bhabha, $\mathrm{H}$.

1994 I luoghi della cultura. Roma: Meltemi.

Forgacs, D. \& Lumley, D.

1996 Italian cultural studies. An introduction. (eds.) Oxford: Oxford University Press.

Galli della Loggia, E. 1998 L'identità italiana. Roma: Il Mulino.

Gangbo, J.

1999 Verso la notte Bakonga. Milano: Lupetti. 
Gangbo, J.

Genette, G.

Genette, G.

Gnisci, A.

Gnisci, A.

Gnisci, A.

Hall, S.

Khouma, P.

Khouma, P.

Khouma, P. \& Ventre, B.

Komla-Ebri, K.

Komla-Ebri, K.
2001 Rometta e Giulieo. Milano: Feltrinelli.

1976 Figure III, Torino: Einaudi.

1987 Seuils. Paris: du Seuil.

1993 Il rovescio del gioco. Roma: Sovera.

1988 La letteratura italiana della migrazione. Roma: Lilith.

2003 Creolizzare l'Europa. Roma: Meltemi.

1997 Old and new identities, old and nex ethnicities. In: King, A.D. (ed.), Culture, globalization and the world system: contemporary conditions for the representation of identity. Minneapolis: University of Minnesota Press.

1990 Io, venditore di elefanti. Una vita per forza tra Dakar, Parigi e Milano. Edited by O. Pivetta. Milano: Garzanti.

2005 Nonno Dio e gli spiriti danzanti. Milano: Baldini Castoldi Dalai.

1994 Nato in Senegal, immigrato in Italia: parlano $i$ senegalesi che vivono nel nostro paese. Milano: Edizioni ambiente.

2002 Imbarazzismi. Quotidiani imbarazzi in bianco e nero. Milano: Edizioni dell'arco.

2002 Neyla. Un incontro due mondi. Milano: Edizioni dell'arco. 
Mauceri, M.C.

Methnani, S. \&

Fortunato, M.

Monteiro Martins, J.

Monteiro Martins, J.

Moussa Ba, S. \& Micheletti, P.A

Parati, G.

Portelli, A.

Remotti, F.

Ricoeur, P.

Scego, I.

Scego, I.

Scego, I.

Scego, I.
2004 Igiaba Scego: la seconda generazione di autori transnazionali sta già emergendo. El Ghibli 4.

1990 Immigrato, Geografie. Roma, Napoli: Theoria.

2000 Racconti italiani. Lecce: Besa.

2005 Quinto seminario degli scrittori migranti.

1991 La promessa di Hamadi, Novara: De Agostini.

2005 Migration Italy. The art of talking back in a destination culture. Toronto Buffalo London: University of Toronto Press.

2006 Fingertips stained with ink. Notes on new migrant writing in Italy. Journal of Postcolonial Studies 8 (3):472-483.

1996 Contro l'identità. Bari: Laterza.

1988 L’identité narrative. Esprit 7-8:295-314.

2003 La nomade che amava Alfred Hitcock. Roma: Sinnos.

2004 Rhoda. Roma: Sinnos.

2005 Italiani per vocazione. Fiesole: Cadmo.

2006 Migranti o vincenti ma scrittori. Dal sito: http:/www.peacelink.it/migranti/ a/15369.html. 
Simone, A.

Tamburri, A.

White, P.
2002 Divenire sans papiers. Sociologia dei dissensi metropolitani. Milano: Mimesis.

1991 To hyphenate or not to hyphenate. The Italian/American writer: an other American. Toronto: Guernica.

1995 Geography, literature and migration. In: Russell King, J.C. \& White, Paul (eds.), Writing across worlds. London \& New York: Routledge. 